Mens

revue d'histoire intellectuelle de l'Amérique française

\title{
Marine Lefèvre. Charles de Gaulle : du Canada français au Québec. Montréal, Leméac, 2007.199p.
}

\section{David Meren}

Volume 10, numéro 1, automne 2009

URI : https://id.erudit.org/iderudit/1023163ar

DOI : https://doi.org/10.7202/1023163ar

Aller au sommaire du numéro

Éditeur(s)

Centre de recherche en civilisation canadienne-française

ISSN

1492-8647 (imprimé)

1927-9299 (numérique)

Découvrir la revue

Citer ce compte rendu

Meren, D. (2009). Compte rendu de [Marine Lefèvre. Charles de Gaulle : $d u$ Canada français au Québec. Montréal, Leméac, 2007. 199 p.] Mens, 10(1),

147-152. https://doi.org/10.7202/1023163ar d'utilisation que vous pouvez consulter en ligne.

https://apropos.erudit.org/fr/usagers/politique-dutilisation/ 
monographie n'avait été réalisée sur le sujet pour la période qui précède la Révolution tranquille. Si, à mon avis, les sources utilisées paraissent parfois trop exclusivement étudiantes et rhétoriques (d'une part, les étudiants sont saisis en vase clos et, d'autre part, l'histoire sociale a été délaissée au profit de l'analyse du discours), il n'en demeure pas moins qu'Impatient d'être soi-même représente une excellente contribution à la littérature qui porte sur les étudiants au Québec. La longueur de la période couverte et l'ambition de mettre en contraste les universités de Montréal et de McGill obligeaient à faire des choix. Ce qu'il nous est donné de lire s'impose comme étant à la fois original et rigoureux. Grâce à cet ouvrage solide, Karine Hébert nous offre une occasion de réfléchir sur le destin des communautés étudiantes qui fait progresser nos analyses.

- Jean-Philippe Warren Département de sociologie et d'anthropologie Université Concordia

\section{Marine Lefèvre. Charles de Gaulle : du Canada français au Québec. Montréal, Leméac, 2007. 199 p.}

Prononcé il y a déjà plus de 40 ans, le "Vive le Québec libre! » de Charles de Gaulle continue de se répercuter. Le dernier écho remonte à la visite du président français Nicolas Sarkozy à Québec en 2008, où certains de ses commentaires suggéraient une rupture avec la politique québécoise du général. L'essai de Marine Lefevre, Charles de Gaulle : du Canada français au Québec, qui est basé sur le mémoire de maîtrise de l'auteure, sonde les origines et l'évolution de cette politique québécoise. L'auteure y examine comment de Gaulle qui, pendant la Seconde Guerre mondiale, considérait le Canada comme "une réussite d'intégration de deux communautés", est devenu un partisan avoué de l'autodétermination du Québec et un critique de la structure fédérale du Canada. Lefevre veut situer cette «rupture» dans l'évolution des relations d'après-guerre de la France 
avec le Canada et le Québec. Elle suggère que les origines de la politique québécoise de De Gaulle résident dans l'éducation et la personnalité du leader français, et dans son idée de la France et dans sa vocation internationale, et dans sa conviction que d'aider le Québec favorisait les objectifs de politique étrangère gaullistes. Lefevvre voit dans le cri lancé du balcon un encouragement, non pas à la souveraineté, mais à l'autodétermination du Québec (mais elle admet que de Gaulle avait des opinions bien précises sur la décision que prendraient et devraient prendre les Québécois). Elle présente les événements dramatiques de juillet 1967 comme un aspect d'une politique qui a mûri au long de plus de trente ans, aboutissant aux efforts de Paris en vue d'obtenir pour le Québec le statut de membre distinct de l'Agence de coopération culturelle et technique (ACCT), la première institution intergouvernementale de la Francophonie.

L'ouvrage débute par une biographie de De Gaulle et une présentation de sa vision du monde. Suit un examen des relations du temps de guerre, quand de Gaulle eut ses premiers contacts substantiels avec le Québec et le Canada. Lefêvre passe ensuite aux relations d'après 1945, examinant le rapprochement officiel FranceQuébec et l'apparition de divergences entre Paris et Québec, d'une part, et Ottawa, d'autre part. Cela mène à une analyse de la visite de De Gaulle en 1967 et de ses conséquences, et notamment à une exploration de la coopération France-Québec à la suite de cette visite, l'accent étant mis plus particulièrement sur la Francophonie.

La principale contribution de l'ouvrage de Lefevrre, au style aéré et accessible, est que l'auteure y situe la politique québécoise de De Gaulle dans un contexte triangulaire plus général. Charles de Gaulle suit ainsi la voie tracée par Renée Lescop (Le pari québécois du général de Gaulle) et Dale Thomson (De Gaulle et le Québec), tout en citant des sources fédérales, québécoises et françaises ne figurant pas dans leurs exposés. Le livre présente une synthèse d'une bonne partie de ce qui a été écrit sur l'histoire du triangle France-Québec-Canada, et Lefèvre fait un tour d'horizon succinct de cette relation en évolution. 
L'ouvrage n'est toutefois pas sans défauts. Ce que Lefevre gagne en brièveté, elle le perd en analyse, de sorte que, si Charles de Gaulle offre généralement une excellente présentation des événements, il n'en propose guère de nouveaux éclairages. Le problème réside beaucoup dans l'approche analytique de Lefevre, qui place le général au centre du récit. L'ouvrage s'appuie sur les mémoires de façon parfois préjudiciable. Lefevre admet qu'il est risqué de tenter de trouver une explication logique ex post facto, mais elle n'en cite pas moins abondamment les Mémoires d'espoir de De Gaulle, publiés en 1970, pour explorer sa vision du Québec et du Canada antérieure à 1967. La distance critique en est amoindrie; ce que disent de Gaulle et d'autres personnes est trop souvent pris au pied de la lettre.

Mais le plus problématique est que les autres acteurs, compte tenu que le texte se concentre sur de Gaulle, ont tendance à ne plus être que des observateurs bidimensionnels, presque passifs. Lefevre fait observer, il faut le reconnaître, que la politique québécoise de De Gaulle constituait dans une importante mesure sa réponse à un Québec en pleine transformation se tournant vers la France. Pourtant, on décèle dans ces propos une vision monolithique du Québec et une acceptation fort peu critique de la conception orthodoxe de la Révolution tranquille. Étant donné que Lefevre reconnaît que de Gaulle était en avance sur l'opinion québécoise, elle aurait pu approfondir davantage le sujet complexe des attitudes québécoises face à la France en général, et aux actions de De Gaulle en particulier. Il n'est par exemple pas fait mention des inquiétudes exprimées par Daniel Johnson à Alain Peyrefitte sur le rythme des événements, lors de la visite du ministre français en septembre 1967. Il n'est question des réticences croissantes du Québec que dans les deux dernières pages de l'ouvrage, ce qui empêche une évaluation plus approfondie de la politique québécoise de De Gaulle.

La dévaluation des autres acteurs s'applique également à la dimension canadienne. L'ouvrage de Lefevvre aurait gagné à s'intéresser davantage à l'historiographie anglophone, ce qui aurait facilité l'exploration du complexe ensemble de préoccupations nationales 
et internationales interdépendantes (et souvent contradictoires) informant les relations d'Ottawa avec la France gaulliste. Nous n'apprenons rien d'important sur ce que pensait de Gaulle de la réaction du gouvernement Pearson à la Révolution tranquille et sur l'influence que cela a pu avoir sur ses actes. Lefevre mentionne le soutien du général à l'égard de la communauté acadienne et reconnaît sa tendance et celles d'autres personnalités françaises à confondre Québec et Canada français, mais elle n'explique pas la place des minorités francophones hors Québec dans la politique gaulliste. Enfin, Lefevre rate une autre occasion d'évaluer d'un œil plus critique les actions de De Gaulle pour le Québec en négligeant d'examiner comment elles ont contribué à l'accession au pouvoir de Pierre Trudeau du fait d'un sentiment de crise au Canada.

En plus de dévaluer d'autres acteurs, l'approche centrée sur de Gaulle tend aussi paradoxalement à rendre plus obscur le sujet qu'elle veut éclaircir. L'obsession de la personnalité minimise des facteurs structurels et culturels plus globaux, de sorte que de Gaulle semble trop souvent se situer au-dessus de l'histoire et hors des tendances générales. Comme de Gaulle n'a pas occupé de fonctions entre 1946 et 1958, ces années ne sont abordées que superficiellement; pourtant, on peut avancer que cette période est cruciale pour comprendre des évènements subséquents. Le différend entre Ottawa et Paris n'est-il apparu qu'après le retour de De Gaulle au pouvoir, comme le laisse entendre Lefevre? Que s'est-il passé au Québec au cours de ces années? Qu'en est-il de l'influence subséquente du "lobby québécois", le groupe de bureaucrates français et de diplomates en poste à Paris au cours des années 1960 qui plaidaient la cause du Québec - cela ne donne-t-il pas à entendre qu'il n'y avait pas que de Gaulle?

Le texte de Lefevre aurait été meilleur à cet égard si l'auteure avait davantage prêté attention à la manière dont la politique québécoise s'insère dans le contexte gaulliste général. Comment envisageait-il l'augmentation de la puissance américaine qui pourrait résulter de l'indépendance du Québec? Philippe de Gaulle nous en donne le seul indice quand il dit que son père croyait que ses actions ne 
détruiraient pas "pour autant» le Canada. Quelle était la place du Québec dans les priorités de Paris dans le cadre de la fondation de l'ACCT (qui n'a eu lieu qu'après le départ de De Gaulle)? Dans quelle mesure Paris agissait-il pour le Québec, et dans quelle mesure ne l'utilisait-il pas plutôt pour promouvoir les intérêts de la France en Afrique? Le cadre national français mériterait aussi une plus grande attention; il est frappant qu'il ne soit pas question de l'influence du rôle de De Gaulle dans la fondation de la Ve République, ni de ses positions relatives à la situation politique du Québec et du Canada, ni non plus de l'incidence du soulèvement politique de Mai 68 sur la politique québécoise.

Finalement, la focalisation sur de Gaulle donne à la dimension politique et intergouvernementale des événements une place disproportionnée. L'éventail des liens privés et quasi publics antérieurs à la Révolution tranquille, qui se sont développés tout au long $\mathrm{du} \mathrm{xx}^{\mathrm{e}}$ siècle, se trouve ainsi ignoré. Lefevre résume souvent simplement des documents officiels sans examiner leur contexte plus général, de sorte que les gouvernements apparaissent trop souvent comme des entités monolithiques; nous n'avons droit, par exemple, qu'à des aperçus, qui nous laissent sur notre faim, de l'hésitation de certains éléments du Quai d'Orsay face aux actions de De Gaulle. La dimension économique n'est mentionnée qu'en passant. Chose plus importante, bien que l'expansion de la coopération culturelle France-Québec soit abordée, le texte de Lefevre aurait été plus riche si l'auteure avait examiné de plus près cette dimension culturelle, et particulièrement le concept de "nation" dans la vision du monde gaullienne et comment il a informé les liens France-Québec. C'est d'autant plus important puisque Lefevre voit dans les facteurs géopolitiques une motivation secondaire de la politique québécoise, et qu'elle affirme que les antécédents de De Gaulle et sa vision de la France le prédisposaient à soutenir le Québec. Une plus grande attention portée à la dimension culturelle aurait aussi poussé l'auteure à examiner comment les notions de solidarité ethnoculturelle favorisaient simultanément rapprochement et malentendu entre de Gaulle et le Québec. 
Lefevre offre une introduction accessible des attitudes et des actions du général à l'égard du Québec qui s'inscrit dans le sillage d'ouvrages précédents. Après avoir refermé Charles de Gaulle, on est cependant frappé par le fait que la focalisation sur le général a limité l'analyse de l'auteure. Lefevre a certes élargi le point de vue au-delà du simple couple Paris-Québec pour s'intéresser aussi à Ottawa, mais elle aurait pu l'élargir encore, favorisant ainsi une compréhension plus profonde non seulement de De Gaulle et de ses attitudes relatives au Québec, mais aussi des trois points du triangle France-QuébecCanada, et de ce que cette relation complexe nous dit sur les relations internationales de la dernière moitié $\mathrm{du} \mathrm{xx}^{\mathrm{e}}$ siècle.

\section{- David Meren \\ London School of Economics et Université de Paris IV-Sorbonne}

Traduction: Christian Bérubé, Musée canadien des civilisations

\section{Jean-Philippe Warren. Une douce anarchie: les années 68 au Québec. Montréal, Boréal, 2008. 311 p.}

La sociologie démystificatrice connaît plusieurs usages: si elle peut rompre le charme des représentations qui contribuent à maintenir en place tel ordre du monde qu'elles présentent comme naturel, elle peut servir, à l'inverse, la maturité désenchantée dont le mot d'ordre est qu'il faut revenir des illusions d'émancipation et des efforts de changer la vie. D'un côté, elle est critique d'un système avec lequel elle irréconcilie; de l'autre, science de la réconciliation avec le monde tel qu'il va. Il n'est pas certain que l'entreprise de JeanPhilippe Warren, malgré la sympathie qu'on lui devine à l'endroit des mouvements politiques radicaux, procède ici du premier type de démystification.

Le mythe qu'Une douce anarchie souhaite remettre en cause est celui d'une génération uniment mobilisée à la fin des années 1960, «levant le poing dans un seul et même élan » et dans "un moment 